\title{
Choroidal findings in idiopathic uveal effusion syndrome
}

This article was published in the following Dove Press journal:

Clinical Ophthalmology

8 November 2011

Number of times this article has been viewed

\section{Tomomi Harada Shigeki Machida \\ Takamistu Fujiwara \\ Yasunori Nishida \\ Dajiro Kurosaka}

Department of Ophthalmology, Iwate Medical University School of Medicine, Iwate, Japan
Correspondence: Shigeki Machida Department of Ophthalmology, Iwate Medical University, School of Medicine, 19-I Uchimaru Morioka Iwate 020-8505, Japan

Tel +8I 19 65I 5III (ext 869I)

Fax +8I 196532864

Email smachida@iwate-med.ac.jp
Purpose: We report choroidal findings by means of enhanced depth imaging spectraldomain optical coherence tomography (EDI-OCT) in a patient with idiopathic uveal effusion syndrome (IUES).

Case report: A 41-year-old man was referred to us with ciliochoroidal and non-rhegmatogenous retinal detachments. Sclerectomies and sclerostomies were performed at the equator in the lower quadrants, resulting in resolution of the ciliochoroidal and retinal detachments. EDI-OCT demonstrated low-reflective areas in the outer choroid. The subfoveal choroidal thickness measured vertically from the outer border of the RPE to the inner border of the sclera was $787 \mu \mathrm{m}$ which was significantly thicker than the normal value $(272 \pm 90 \mu \mathrm{m}, \mathrm{n}=131)$ obtained from age-matched normal controls.

Conclusions: The findings made by EDI-OCT have provided additional evidence that choroidal alterations play a role in the pathological process in IUES.

Keywords: uveal effusion syndrome, spectral-domain optical coherence tomography, EDI-OCT, OCT, choroid

\section{Introduction}

Idiopathic uveal effusion syndrome (IUES) is a rare disease characterized by ciliochoroidal and non-rhegmatogenous retinal detachments associated with an abnormality of trans-scleral diffusion of extravascular proteins in the choroid. ${ }^{1,2}$ Therefore, enhancement of protein diffusion by lamellar sclerectomies and sclerostomies results in resolution of the ciliochoroidal and non-rhegmatogenous retinal detachments. ${ }^{3}$

Enhanced depth imaging spectral-domain optical coherence tomography (EDIOCT) has been developed to allow clinicians to evaluate the thickness and structure of the choroid. ${ }^{4}$ For example, it has been demonstrated in EDI-OCT images that choroidal thickness is significantly thinner in highly myopic eyes. ${ }^{5}$

We present our EDI-OCT findings together with the choroidal angiographic findings in a patient with IUES.

\section{Case report}

A 41-year-old man was referred to us in July 2001 complaining of decreased vision in his right eye. He had a history of serous macular detachments in both eyes in 1996, which resolved spontaneously. Otherwise, he was healthy. His best-corrected visual acuity (BCVA) was 0.3 OD and 1.0 OS with mild myopia in both eyes. The intraocular pressures were normal. The axial lengths of the globe were $22.95 \mathrm{~mm}$ and $22.65 \mathrm{~mm}$ for the right and left eyes, respectively. There were no inflammatory cells in the anterior 
chamber and vitreous cavity in either eyes. The posterior pole of the ocular fundus showed atrophy of the retinal pigment epithelium (RPE; Figure 1A). An annular ciliochoroidal detachment and a shifting bullous retinal detachment were noted in the right eye (Figure 1B and C). In the left eye, a ciliochoroidal detachment was seen in the temporal hemisphere without retinal detachment. The vortex veins were only discernible in the temporal superior quadrant in the right eye and in the inferior lower quadrant in the left eye.

Sclerectomies and sclerostomies were performed at the equator in the lower quadrants of the right eye in September 2001, resulting in resolution of the ciliochoroidal and retinal detachments. During the surgery, we noted that the sclera was rigid and thick. The ciliochoroidal and retinal detachments recurred 6 months after the surgery, and the vision decreased to light perception in the right eye because of chorioretinal atrophy.

The patient re-visited us complaining of decreased vision in the left eye, in September 2005. The BCVA was light perception OD and 0.3 OS. Atrophy of the RPE in the posterior pole and annular ciliochoroidal detachments were noted in the left eye (Figure 1D and E). OCT demonstrated subretinal fluid in the macula (Figure 1F). We performed sclerectomies

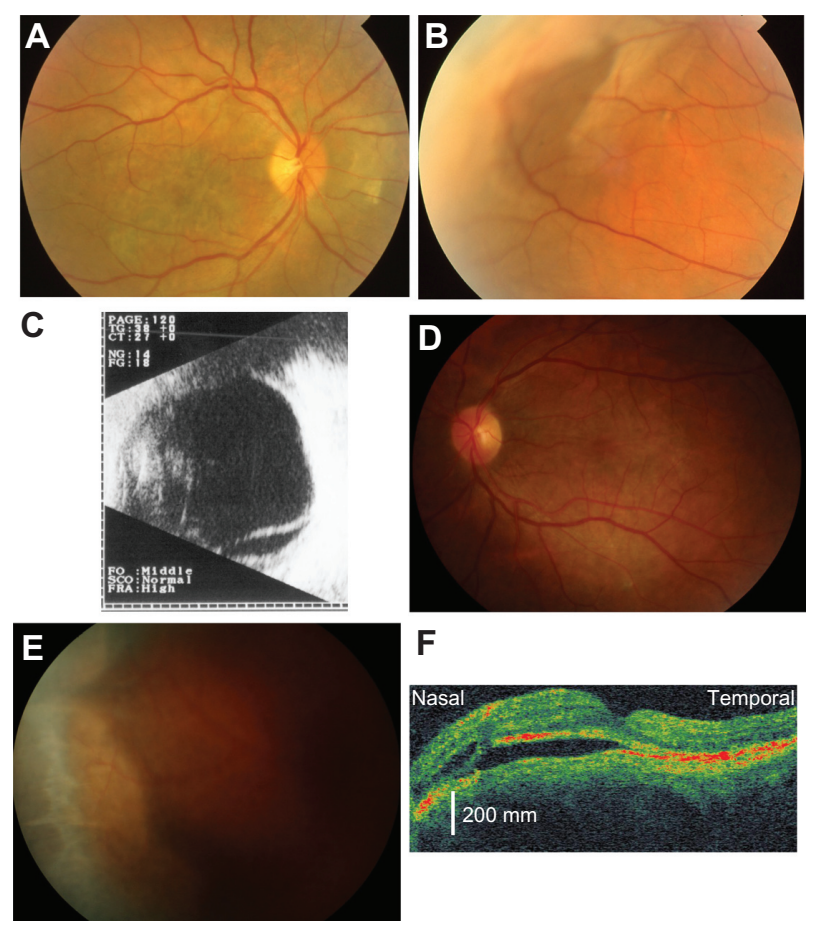

Figure I Photograph of the posterior pole (A) and peripheral region (B) of the right eye of a patient with idiopathic uveal effusion syndrome (IUES) in July 2001. A choroidal detachment can be seen (B). The ultrasonographic image of the right eye shows a retinal detachment in the inferior hemisphere (C). Photograph of the posterior pole (D) and peripheral region (E) of the left eye in September 2006. A ciliochoroidal detachment can be seen (E). Optical coherence tomography shows a macular detachment in the left eye $(\mathbf{F})$. and sclerostomies with application of mitomycin C (MMC) in the inferior quadrants of the left eye in November 2005. Medical Quick Absorber ${ }^{\circledR}$ (Inami Co, Tokyo, Japan) soaked in $0.04 \%$ MMC was placed on the sclera after the sclerostomies for 5 minutes to avoid postoperative fibrosis as used for glaucoma filtering surgery. The ciliochoroidal and retinal detachments promptly resolved after surgery with improvement of vision to 1.0 .

The patient returned because of a relapse in the left eye in March 2010. His BCVA had deteriorated to 0.7 OS. OCT demonstrated a serous macular detachment and intraretinal fluid.

In May 2010, fluorescein angiography (FAG) showed marked hyperfluorescence in the macular region of the left eye due to window defect with late subretinal leakage (Figure 2A and B). Indocyanine green angiography (ICGA) revealed diffuse and moderate hyperfluorescence that obscured large choroidal veins in the posterior pole of the ocular fundus in the early phase (Figure 2C), which was consistent with previously reported ICGA findings. ${ }^{6}$ In the late phase of ICGA, hypofluorescence was noted throughout the posterior pole (Figure 2D). Topical steroid application resulted in a resolution of the serous macular detachment and the intraretinal fluid in the left eye, with improvement in vision.
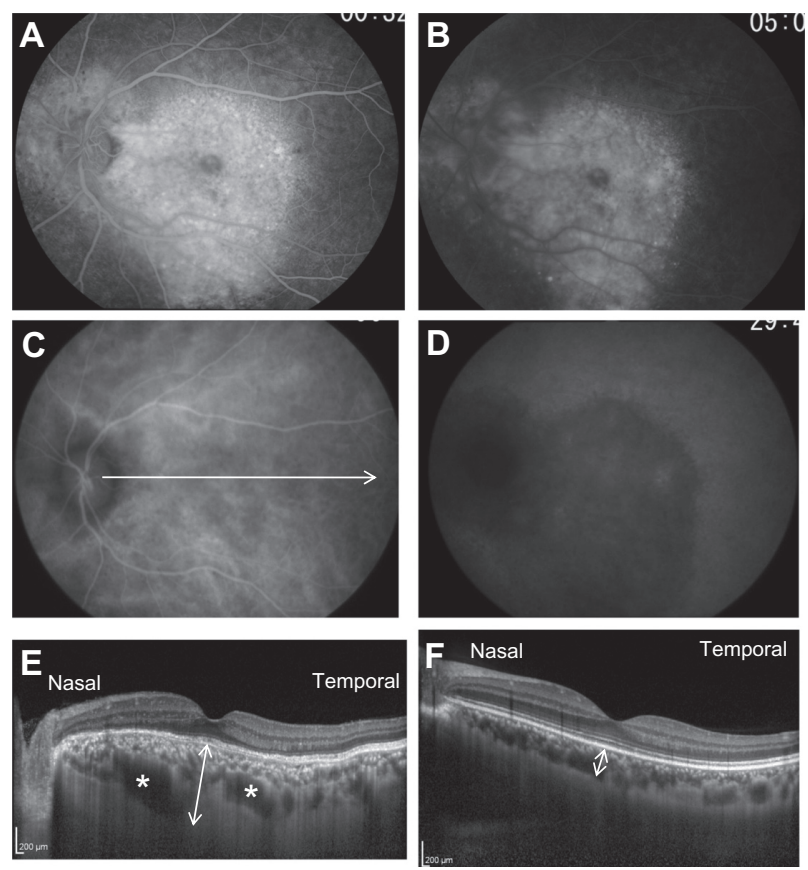

Figure 2 Fluorescein angiograms (A and $\mathbf{B})$ and indocyanine green angiograms (C and D) in May 2008. The arrow points to the direction and length of the enhanced depth imaging optical coherence tomographic scan (EDI-OCT) (C). The EDI-OCT image shows large low-reflective areas (indicated by asterisks) in the outer choroid in the patient (E) which was not noted in a control subject $(\mathbf{F})$. 
The arrow and line in Figure $2 \mathrm{C}$ indicates the direction and length of the EDI-OCT scan. EDI-OCT demonstrated low-reflective areas (indicated by asterisks in Figure 2E) in the outer choroid (Figure 2C and E) in April 2011. The subfoveal choroidal thickness was measured vertically from the outer border of the RPE to the inner border of the sclera. The choroidal thickness was $787 \mu \mathrm{m}$ (indicated by the arrow in Figure 2E) which was significantly thicker than the normal value $(272 \pm 90 \mu \mathrm{m}, \mathrm{n}=131)$ obtained from age-matched normal controls (indicated by the arrow in Figure 2F).

\section{Discussion}

Our patient had annular ciliochoroidal detachments with shifting subretinal fluid which was resolved by the sclerectomies and sclerostomies. The patient was a healthy and middle-aged man without nanophthalmos or medical history of any other disease. These characteristics are consistent with those of IUES. ${ }^{2}$

The EDI-OCT images showed large low-reflective areas in the outer choroid, which could represent dilated choroidal veins or an enlargement of the suprachoroidal space. The ICGA failed to delineate large choroidal veins in the posterior pole in the early phase because of the diffuse hyperfluorescence throughout the ocular fundus. However, congestion of the choroidal veins could take place because of the abnormality of the vortex veins. Alternatively, the low-reflective areas in the EDI-OCT images may represent an accumulation of extravascular proteins in the suprachoroidal space. These choroidal findings are comparable to the hypothesized disease mechanism of IUES in which extravascular proteins triggered by choroidal congestion accumulate in the suprachoroidal space leading to choroidal and retinal detachments. ${ }^{3}$ A congenital anomaly of the sclera superimposed on aging and hormonal changes is hypothesized to prevent transport of extravascular proteins across the sclera. ${ }^{3}$ In fact, the sclera of our patient appeared to be thick and rigid during the surgeries.

The findings made by EDI-OCT have provided additional evidence that choroidal alterations play a role in the pathological process in IUES.

\section{Disclosure}

The authors report no conflicts of interest in this work.

\section{References}

1. Schepens CL, Brockhurst RJ. Uveal effusion. I. Clinical picture. Arch Ophthalmol. 1963;70:189-201.

2. Gass JDM, Jallow S. Idiopathic serous detachment of the choroid, ciliary body, and retina (uveal effusion syndrome). Ophthalmology. 1982;89(9):1018-1032.

3. Gass JDM. Uveal effusion syndrome. A new hypothesis concerning pathogenesis and technique of surgical treatment. Retina. 1983;3(3): 159-163.

4. Spaide RF, Koizumi H, Pozonni MC. Enhanced depth imaging spectral-domain optical coherence tomography. Am J Ophthalmol. 2008;146(4):496-500.

5. Fujiwara T, Imamura Y, Margolis R, Slakter JS, Spaide RF. Enhanced depth imaging optical coherence tomography of the choroid in highly myopic eyes. Am J Ophthalmol. 2009;148(3):445-450.

6. Uyama M, Takahashi K, Kozaki J, et al. Uveal effusion syndrome: clinical features, surgical treatment, histologic examination of the sclera, and pathophysiology. Ophthalmology. 2000;107(3):441-449.
Clinical Ophthalmology

\section{Publish your work in this journal}

Clinical Ophthalmology is an international, peer-reviewed journal covering all subspecialties within ophthalmology. Key topics include: Optometry; Visual science; Pharmacology and drug therapy in eye diseases; Basic Sciences; Primary and Secondary eye care; Patient Safety and Quality of Care Improvements. This journal is indexed on

\section{Dovepress}

PubMed Central and CAS, and is the official journal of The Society of Clinical Ophthalmology (SCO). The manuscript management system is completely online and includes a very quick and fair peer-review system, which is all easy to use. Visit http://www.dovepress.com/ testimonials.php to read real quotes from published authors. 